\title{
Method of field expansions for vector electromagnetic scattering by layered periodic crossed gratings
}

\author{
David P. Nicholls \\ Department of Mathematics, Statistics, and Computer Science, University of Illinois at Chicago, Chicago, \\ Illinois 60607,USA (davidn@uic.edu)
}

Received December 23, 2014; revised February 19, 2015; accepted February 19, 2015; posted February 20, 2015 (Doc. ID 231273); published April 6, 2015

\begin{abstract}
In many applications of scientific and engineering interest the accurate modeling of scattering of linear waves by periodic layered media plays a crucial role. From geophysics and oceanography to materials science and imaging, the ability to simulate such configurations numerically in a rapid and robust fashion is of paramount importance. In this contribution we focus upon the specific problem of vector electromagnetic radiation interacting with a multiply layered periodic crossed diffraction grating. While all of the classical methods for the numerical simulation of partial differential equations have been brought to bear upon this problem, we argue here that in this particular context a high-order perturbation of surfaces approach is superior. In particular, we describe how the method of field expansions can be extended to the fully vectorial and three-dimensional scattering problem in the presence of multiple layers. With specific numerical experiments we will show the remarkable efficiency, fidelity, and high-order accuracy one can achieve with an implementation of this algorithm. (c) 2015 Optical Society of America
\end{abstract}

OCIS codes: (050.1755) Computational electromagnetic methods; (240.6680) Surface plasmons.

http://dx.doi.org/10.1364/JOSAA.32.000701

\section{INTRODUCTION}

The scattering of linear waves by periodic layered media plays a central role in applications of wide scientific and engineering interest. From geophysics [1] and oceanography [2] to materials science [3] and imaging [ $\underline{4}$ ], the ability to simulate such configurations numerically in a rapid and robust fashion is of paramount importance. In this contribution we focus upon the specific problem of vector electromagnetic radiation interacting with a multiply layered periodic crossed diffraction grating (see Fig. 4). A crossed grating is usually defined as two line gratings at right angles to each other, and we model this with a biperiodic layer interface (which permits nonorthogonal line intersection). We further allow for several such interfaces provided that they all have a common period cell. This model arises in a wide range of applications in optics and photonics, particularly, in regard to the algorithm advocated here, in nanoplasmonics [ $\underline{5}-\underline{7}]$, where one can investigate topics as diverse as extraordinary optical transmission [8], surface enhanced spectroscopy [9], and surface plasmon resonance biosensing [ $\underline{10}-15]$.

While all of the classical numerical algorithms have been brought to bear upon this problem, each faces challenges. The most widely used methods are those based upon finite elements (see, e.g., [16-19]) and finite differences (see, e.g., [20-22]). However, these volumetric approaches are clearly disadvantaged with an unnecessarily large number of unknowns for the problem at hand which features piecewiseconstant dielectric constants. Additionally, in such schemes the unbounded problem domain must be truncated at some finite distance from the grating structure inducing the complication and error introduced by enforcing an (approximately) nonreflecting boundary condition (e.g., the perfectly matched layer of Berenger [23] and variants of this, e.g., [24-26]).
Methods based upon traditional integral equation (IE) formulations [27] are a natural candidate but face several challenges. First, specially designed quadrature rules must be designed to deliver high-order (spectral) accuracy. Second, such rules applied to these nonlocal IEs generate dense, nonsymmetric positive definite linear systems to be solved. However, these issues have been adequately addressed (possibly with the use of iterative solution procedures accelerated by fast multipole methods [28]), and they are a compelling alternative (see, e.g., the survey article [29] for more details). However, three properties render them noncompetitive for the periodic, parametrized problems we consider as compared with the methods we advocate here:

1. For periodic problems the relevant Greens function must be periodized if one is to restrict the domain of integration to a single period cell. This is a well-known problem (see, e.g., the introduction of [30] for a full description), and the slow convergence of the periodization must be accelerated (e.g., with techniques such as Ewald summation). However, even with such technology, these IE methods demand an additional discretization parameter: The number of terms retained in the approximation of the periodized Greens function.

2. For configurations parametrized by the real value $\varepsilon$ (for us the height/slope of the crossed interface), an IE solver will return the scattering returns only for a particular value of $\varepsilon$. If this value is changed then the solver must be run again.

3. The dense, nonsymmetric positive definite systems of linear equations which must be inverted with each simulation.

As an alternative we advocate here for a "high-order perturbation of surfaces" (HOPS) algorithm, more specifically the method of field expansions (FE) appropriately generalized to the fully three-dimensional vector Maxwell equations posed 
on a multiply layered medium. These schemes trace their roots to the low-order calculations of Rayleigh [31] and Rice [32]. Their high-order incarnation for doubly layered media was first introduced by Bruno and Reitich for the two-dimensional scalar case in $[33,34]$ and for the fully three-dimensional vector Maxwell case in [35]. These were further enhanced and stabilized by the author and Reitich [36-38], and expanded to multiple layers in the two-dimensional scalar case by the author and Malcolm [39].

These formulations are particularly compelling as they maintain the advantageous properties of classical IE formulations (e.g., surface formulation and exact enforcement of farfield conditions) while avoiding the shortcomings listed above:

1. As this HOPS scheme utilizes the eigenfunctions of the Laplacian (suitable complex exponentials) on a periodic domain, the quasiperiodicity of solutions is built in and does not need to be further approximated.

2. Since the methods are built upon expansions in the boundary parameter, $\varepsilon$, once the Taylor coefficients are known for the scattering quantities, it is simply a matter of summing these (rather than beginning a new simulation) for any given choice of $\varepsilon$ to recover the returns.

3. Due to the perturbative nature of the scheme, at every perturbation order one need only invert a single, sparse operator corresponding to the flat-interface approximation of the problem.

The rest of the paper is organized as follows: In Section 2 we briefly recall the equations which govern the propagation of electromagnetic waves in a periodic, three-dimensional multiply layered structure. In Section 3 we specify the method of FE for numerically approximating solutions to these governing equations, in particular the generalizations of [35] and [39] necessary for multiple layers for the vector Maxwell equations. In Section 4 we detail our numerical results, including a convergence study in Section 4.C and simulations of surface plasmon resonances (SPRs) in Section 4.D.

\section{GOVERNING EQUATIONS}

Consider a layered diffraction grating with $M$ many crossed periodic interfaces located at

$$
z=\bar{g}^{(m)}+g^{(m)}(x, y), \quad 1 \leq m \leq M,
$$

where $z$ is the vertical coordinate and $x$ and $y$ are the lateral coordinates, which delineate $(M+1)$ many layers,

$$
S^{(0)}:=\left\{z>\bar{g}^{(1)}+g^{(1)}(x, y)\right\},
$$

and, for $1 \leq m \leq M-1$,

$$
S^{(m)}:=\left\{\bar{g}^{(m+1)}+g^{(m+1)}(x, y)<z<\bar{g}^{(m)}+g^{(m)}(x, y)\right\},
$$

and

$$
S^{(M)}:=\left\{z<\bar{g}^{(M)}+g^{(M)}(x, y)\right\}
$$

Each layer is filled by with a material having dielectric constant $\epsilon^{(m)}$, and the permeability of each is equal to $\mu_{0}$, that of the vacuum. In this contribution we consider the genuinely three-dimensional setting where the grating interfaces are crossed and periodic, $g^{(m)}\left(x+d_{1}, y\right)=g^{(m)}(x, y), \quad g^{(m)}\left(x, y+d_{2}\right)=g^{(m)}(x, y)$,

for $1 \leq m \leq M$.

The structure is illuminated from above by plane-wave incidence of the form

$$
\begin{aligned}
& \mathbf{E}^{\mathrm{inc}}(x, y, z):=e^{i \omega t} \underline{\mathbf{E}}^{\mathrm{inc}}(x, y, z, t)=\mathbf{A} e^{i(\alpha x+\beta y-\gamma z)} \\
& \mathbf{H}^{\text {inc }}(x, y, z):=e^{i \omega t} \underline{\mathbf{H}}^{\text {inc }}(x, y, z, t)=\mathbf{B} e^{i(\alpha x+\beta y-\gamma z)} \text {, }
\end{aligned}
$$

where

$$
\mathbf{A} \cdot \kappa=0, \quad \mathbf{B}=\frac{1}{\omega \mu_{0}} \kappa \times \mathbf{A}, \quad|\mathbf{A}|=|\mathbf{B}|=1,
$$

and $\kappa:=(\alpha, \beta,-\gamma)^{T}$.

The time-harmonic Maxwell equations for the reduced total fields can be written (upon dropping the harmonic factor $\exp (-i \omega t))$ as

$$
\begin{array}{ll}
\nabla \times \mathbf{E}=i \omega \mu_{0} \mathbf{H}, & \operatorname{div}[\mathbf{E}]=0, \\
\nabla \times \mathbf{H}=-i \omega \epsilon \mathbf{E}, & \operatorname{div}[\mathbf{H}]=0 .
\end{array}
$$

All fields satisfy the vector Helmholtz equation, for instance,

$$
\Delta \mathbf{E}+k^{2} \mathbf{E}=0,
$$

with $k^{2}=\omega^{2} \epsilon \mu_{0}$, which encodes the properties of the material and the frequency of radiation into one constant.

We decompose the total fields into incident and scattered components by

$$
\begin{gathered}
\mathbf{E}=\left\{\begin{array}{cc}
\mathbf{E}^{(0)}+\mathbf{E}^{\text {inc }} & m=0 \\
\mathbf{E}^{(m)} & 1 \leq m \leq M
\end{array}\right. \\
\mathbf{H}=\left\{\begin{array}{cc}
\mathbf{H}^{(0)}+\mathbf{H}^{\text {inc }} & m=0 \\
\mathbf{H}^{(m)} & 1 \leq m \leq M
\end{array},\right.
\end{gathered}
$$

and note that each of these must satisfy vector Helmholtz equations; for example, for $0 \leq m \leq M$,

$$
\Delta \mathbf{E}^{(m)}+\left(k^{(m)}\right)^{2} \mathbf{E}^{(m)}=0, \quad \text { in } S^{(m)},
$$

where $\left(k^{(m)}\right)^{2}:=\omega^{2} \epsilon^{(m)} \mu_{0}$ and $\alpha^{2}+\beta^{2}+\gamma^{2}=\left(k^{(0)}\right)^{2}$.

At the material interfaces the fields are coupled by the transmission conditions, for $1 \leq m \leq M$,

$$
\begin{aligned}
& N^{(m)} \times\left[\mathbf{E}^{(m-1)}-\mathbf{E}^{(m)}\right]=\zeta^{(m)}, \\
& N^{(m)} \times\left[\mathbf{H}^{(m-1)}-\mathbf{H}^{(m)}\right]=\psi^{(m)},
\end{aligned}
$$

where $N^{(m)}:=\left(\begin{array}{lll}-\partial_{x} g^{(m)} & -\partial_{y} g^{(m)} & 1\end{array}\right)^{T}$, and, in the present context of plane-wave illumination from above,

$$
\begin{gathered}
\zeta^{(1)}=-N^{(1)} \times\left[\mathbf{E}^{\text {inc }}\right]_{z=\bar{g}^{(1)}+g^{(1)},} \\
\psi^{(1)}=-N^{(1)} \times\left[\mathbf{H}^{\text {inc }}\right]_{z=\bar{g}^{(1)}+g^{(1)},}
\end{gathered}
$$

and $\zeta^{(m)} \equiv \psi^{(m)} \equiv 0,2 \leq m \leq M$. In light of Maxwell's equations, Eqs. (2.1), we can rewrite this in terms of the electric field as 


$$
\begin{gathered}
N^{(m)} \times\left[\mathbf{E}^{(m-1)}-\mathbf{E}^{(m)}\right]_{z=\bar{g}^{(m)}+g^{(m)}}=\zeta^{(m)}, \\
N^{(m)} \times\left[\nabla \times\left[\mathbf{E}^{(m-1)}-\mathbf{E}^{(m)}\right]\right]_{z=\bar{g}^{(m)}+g^{(m)}}=\psi^{(m)}, \quad 1 \leq m \leq M,
\end{gathered}
$$

where $\psi^{(1)}=-N^{(1)} \times\left[\nabla \times\left[\mathbf{E}^{\text {inc }}\right]\right]_{z=\bar{g}^{(1)}+g^{(1)}}$.

Finally, the periodicity of the grating interfaces implies that the fields are quasiperiodic, for example,

$$
\mathbf{E}^{(m)}\left(x+d_{1}, y+d_{2}, z\right)=e^{i\left(\alpha d_{1}+\beta d_{2}\right)} \mathbf{E}^{(m)}(x, y, z),
$$

and we demand that $\mathbf{E}^{(0)}$ and $\mathbf{E}^{(M)}$ be outgoing at positive and negative infinity, respectively.

\section{A. Rayleigh Expansions}

Separation of variables gives the Rayleigh expansions, which are quasiperiodic, outgoing solutions of Eq. (2.2). The electric fields can be written

$$
\mathbf{E}^{(0)}(x, y, z)=\sum_{p=-\infty}^{\infty} \sum_{q=-\infty}^{\infty} \mathbf{a}_{p, q}^{(0)} e^{i \gamma_{p, q}^{(0)}\left(z-\bar{g}^{(1)}\right)} e^{i\left(\alpha_{p} x+\beta_{q} y\right)}
$$

and, for $1 \leq m \leq M-1$,

$$
\begin{aligned}
\mathbf{E}^{(m)}(x, y, z)= & \sum_{p=-\infty}^{\infty} \sum_{q=-\infty}^{\infty}\left\{\mathbf{d}_{p, q}^{(m)} \frac{\sinh \left(i \gamma_{p, q}^{(m)}\left(z-\bar{g}^{(m+1)}\right)\right)}{\sinh \left(i \gamma_{p, q}^{(m)}\left(\bar{g}^{(m)}-\bar{g}^{(m+1)}\right)\right)}\right. \\
& \left.+\mathbf{a}_{p, q}^{(m)} \frac{\sinh \left(i \gamma_{p, q}^{(m)}\left(\bar{g}^{(m)}-z\right)\right)}{\sinh \left(i \gamma_{p, q}^{(m)}\left(\bar{g}^{(m)}-\bar{g}^{(m+1)}\right)\right)}\right\} e^{i\left(\alpha_{p} x+\beta_{q} y\right)},
\end{aligned}
$$

and

$$
\mathbf{E}^{(M)}(x, y, z)=\sum_{p=-\infty}^{\infty} \sum_{q=-\infty}^{\infty} \mathbf{d}_{p, q}^{(M)} e^{i \gamma_{p, q}^{(M)}\left(\bar{g}^{(M)}-z\right)} e^{i\left(\alpha_{p} x+\beta_{q} y\right)},
$$

where, for $p, q \in \mathbf{Z}, 0 \leq m \leq M$,

$$
\begin{array}{r}
\alpha_{p}:=\alpha+\left(2 \pi / d_{1}\right) p, \quad \beta_{q}:=\beta+\left(2 \pi / d_{2}\right) q, \\
\gamma_{p, q}^{(m)}:=\left\{\begin{array}{cc}
\sqrt{\left(k^{(m)}\right)^{2}-\alpha_{p}^{2}-\beta_{q}^{2}} & (p, q) \in \mathcal{U}^{(m)} \\
i \sqrt{\alpha_{p}^{2}+\beta_{q}^{2}-\left(k^{(m)}\right)^{2}} & (p, q) \notin \mathcal{U}^{(m)}
\end{array}\right.
\end{array}
$$

and

$$
\mathcal{U}^{(m)}=\left\{p, q \in \mathbf{Z} \mid \alpha_{p}^{2}+\beta_{q}^{2}<\left(k^{(m)}\right)^{2}\right\},
$$

which are the propagating modes in the uppermost and lowest layers $\left(S^{(0)}\right.$ and $S^{(M)}$, respectively). We point out that $\mathbf{a}_{p, q}^{(0)}$ and $\mathbf{d}_{p, q}^{(M)}$ are the upward and downward propagating Rayleigh amplitudes. Quantities of great interest are the efficiencies

$$
e_{p, q}^{(0)}=\left(\gamma_{p, q}^{(0)} / \gamma\right)\left|\mathbf{a}_{p, q}^{(0)}\right|^{2}, \quad e_{p, q}^{(M)}=\left(\gamma_{p, q}^{(M)} / \gamma\right)\left|\mathbf{d}_{p, q}^{(M)}\right|^{2},
$$

and the object of fundamental importance to the design of SPR biosensors [10-15] is the "reflectivity map,"

$$
R:=\sum_{(p, q) \in \mathcal{U}^{(0)}} e_{p, q}^{(0)} .
$$

If $M=1$ and layer $M$ is filled with a perfect electric conductor, then, if a lossless dielectric fills $S^{(0)}$, conservation of energy requires that $R=1$. As we shall see (Section 4.D), this is not the case for a metal (such as gold) in the lower domain and drops in its value to a 10th or even a 100th are the fundamental phenomena behind the utility of these sensors.

\section{FIELD EXPANSIONS}

In essence, the method of FE [33-35] is a perturbative approach to enforcing the boundary conditions of Eqs. (2.3a) and (2.3b) with the $\left\{\mathbf{a}_{p, q}^{(m)}, \mathbf{d}_{p, q}^{(m)}\right\}$ from the Rayleigh expansions, Eq. (2.4), as unknowns. Here we take a slightly different (but equivalent) point of view which, we believe, simplifies the presentation, particularly in the vector-valued context of the Maxwell equations. First, we define the following functions:

$$
\mathbf{a}^{(0)}(x, y):=\mathbf{E}^{(0)}\left(x, y, \bar{g}^{(1)}\right)=\sum_{p=-\infty}^{\infty} \sum_{q=-\infty}^{\infty} \mathbf{a}_{p, q}^{(0)} e^{i\left(\alpha_{p} x+\beta_{q} y\right)},
$$

and, for $1 \leq m \leq M-1$,

$$
\mathbf{d}^{(m)}(x, y):=\mathbf{E}^{(m)}\left(x, y, \bar{g}^{(m)}\right)=\sum_{p=-\infty}^{\infty} \sum_{q=-\infty}^{\infty} \mathbf{d}_{p, q}^{(m)} e^{i\left(\alpha_{p} x+\beta_{q} y\right)},
$$

and, for $1 \leq m \leq M-1$,

$$
\mathbf{a}^{(m)}(x, y):=\mathbf{E}^{(m)}\left(x, y, \bar{g}^{(m+1)}\right)=\sum_{p=-\infty}^{\infty} \sum_{q=-\infty}^{\infty} \mathbf{a}_{p, q}^{(m)} e^{i\left(\alpha_{p} x+\beta_{q} y\right)},
$$

and

$$
\mathbf{d}^{(M)}(x, y):=\mathbf{E}^{(M)}\left(x, y, \bar{g}^{(M)}\right)=\sum_{p=-\infty}^{\infty} \sum_{q=-\infty}^{\infty} \mathbf{d}_{p, q}^{(M)} e^{i\left(\alpha_{p} x+\beta_{q} y\right)},
$$

which are the "flat interface" field traces.

Remark. An important point is that these unknowns belong to a space of three-functions, $\mathcal{F}^{3}$, by which we mean that they map two dependent variables, $(x, y)$, to three components, for instance, $\mathbf{a}^{(m)}: \mathbf{R}^{2} \rightarrow \mathbf{R}^{3}$. In Eq. (3.4) we will explicitly enforce that these be divergence free.

\section{A. Surface Operators}

As our method will be focused upon the interfacial boundary conditions, Eq. (2.3), we note that only four of these six are linearly independent. We will fix upon the $x$ - and $y$-components of Eqs. (2.3a) and $(\underline{2.3 \mathrm{~b}})$ and therefore define the projection operator

$$
\mathbf{P}_{x, y}\left[\left(\begin{array}{c}
v^{x} \\
v^{y} \\
v^{z}
\end{array}\right)\right]:=\left(\begin{array}{c}
v^{x} \\
v^{y}
\end{array}\right), \quad \mathbf{P}_{x, y}: \mathcal{F}^{3} \rightarrow \mathcal{F}^{2}
$$

With this we further define the Dirichlet trace operators,

$$
\mathcal{D}(0): \mathbf{a}^{(0)} \rightarrow \mathbf{P}_{x, y}\left[N^{(1)} \times \mathbf{E}^{(0)}\right]_{z=\bar{g}^{(1)}+g^{(1)},}
$$


and, for $1 \leq m \leq M-1$

$$
\mathcal{D}(m):\left(\begin{array}{c}
\mathbf{d}^{(m)} \\
\mathbf{a}^{(m)}
\end{array}\right) \rightarrow\left(\begin{array}{c}
\mathbf{P}_{x, y}\left[N^{(m)} \times \mathbf{E}^{(m)}\right]_{z=\bar{g}^{(m)}+g^{(m)}} \\
\mathbf{P}_{x, y}\left[N^{(m+1)} \times \mathbf{E}^{(m)}\right]_{z=\bar{g}^{(m+1)}+g^{(m+1)}}
\end{array}\right),
$$

and

$$
\mathcal{D}(M): \mathbf{d}^{(M)} \rightarrow \mathbf{P}_{x, y}\left[N^{(M)} \times \mathbf{E}^{(M)}\right]_{z=\bar{g}^{(M)}+g^{(M)} .}
$$

We also define their (exterior) Neumann counterparts,

$$
\mathcal{N}(0): \mathbf{a}^{(0)} \rightarrow \mathbf{P}_{x, y}\left[N^{(1)} \times \nabla \times\left[\mathbf{E}^{(0)}\right]\right]_{z=\bar{g}^{(1)}+g^{(1)},}
$$

and for $1 \leq m \leq M-1$,

$$
\mathcal{N}(m):\left(\begin{array}{c}
\mathbf{d}^{(m)} \\
\mathbf{a}^{(m)}
\end{array}\right) \rightarrow\left(\begin{array}{c}
\mathbf{P}_{x, y}\left[N^{(m)} \times \nabla \times\left[\mathbf{E}^{(m)}\right]\right]_{z=\bar{g}^{(m)}+g^{(m)}} \\
\mathbf{P}_{x, y}\left[N^{(m+1)} \times \nabla \times\left[\mathbf{E}^{(m)}\right]\right]_{z=\bar{g}^{(m+1)}+g^{(m+1)}}
\end{array}\right),
$$

and

$$
\mathcal{N}(M): \mathbf{d}^{(M)} \rightarrow \mathbf{P}_{x, y}\left[N^{(M)} \times \nabla \times\left[\mathbf{E}^{(M)}\right]\right]_{z=\bar{g}^{(M)}+g^{(M)}} .
$$

The idea behind these operators $\mathcal{D}$ and $\mathcal{N}$ is that they map, respectively, the function pair $\left(\mathbf{d}^{(m)}, \mathbf{a}^{(m)}\right)$ to the upper and lower Dirichlet and Neumann traces. For later convenience we write, for $1 \leq m \leq M-1$,

$$
\mathcal{D}(m)=\left(\begin{array}{ll}
\mathcal{D}_{(m)}^{u, d} & \mathcal{D}_{(m)}^{u, a} \\
\mathcal{D}_{(m)}^{\ell, d} & \mathcal{D}_{(m)}^{\ell, a}
\end{array}\right), \quad \mathcal{N}(m)=\left(\begin{array}{cc}
\mathcal{N}_{(m)}^{u, d} & \mathcal{N}_{(m)}^{u, a} \\
\mathcal{N}_{(m)}^{\ell, d} & \mathcal{N}_{(m)}^{\ell, a}
\end{array}\right)
$$

To keep the notation consistent we denote

$$
\begin{array}{clrl}
\mathcal{D}(0) & =\mathcal{D}_{(0)}^{\ell, a}, & \mathcal{N}(0)=\mathcal{N}_{(0)}^{\ell, a}, \\
\mathcal{D}(M)=\mathcal{D}_{(M)}^{u, d}, & & \mathcal{N}(M)=\mathcal{N}_{(M)}^{u, d} .
\end{array}
$$

In terms of these, the Dirichlet boundary conditions, Eq. (2.3a), become

$$
\mathcal{D}_{(0)}^{\ell, a}\left[\mathbf{a}^{(0)}\right]-\mathcal{D}_{(1)}^{u, d}\left[\mathbf{d}^{(1)}\right]-\mathcal{D}_{(1)}^{u, a}\left[\mathbf{a}^{(1)}\right]=\zeta^{(1)},
$$

and, for $2 \leq m \leq M-1$,

$\mathcal{D}_{(m-1)}^{\ell, d}\left[\mathbf{d}^{(m-1)}\right]+\mathcal{D}_{(m-1)}^{\ell, a}\left[\mathbf{a}^{(m-1)}\right]-\mathcal{D}_{(m)}^{u, d}\left[\mathbf{d}^{(m)}\right]-\mathcal{D}_{(m)}^{u, a}\left[\mathbf{a}^{(m)}\right]=\zeta^{(m)}$,

and

$$
\mathcal{D}_{(M-1)}^{\ell, d}\left[\mathbf{d}^{(M-1)}\right]+\mathcal{D}_{(M-1)}^{\ell, a}\left[\mathbf{a}^{(M-1)}\right]-\mathcal{D}_{(M)}^{u, d}\left[\mathbf{d}^{(M)}\right]=\zeta^{(M)} .
$$

The Neumann conditions, Eq. (2.3b), become

$$
\mathcal{N}_{(0)}^{\ell, a}\left[\mathbf{a}^{(0)}\right]-\mathcal{N}_{(1)}^{u, d}\left[\mathbf{d}^{(1)}\right]-\mathcal{N}_{(1)}^{u, a}\left[\mathbf{a}^{(1)}\right]=\psi^{(1)},
$$

and, for $2 \leq m \leq M-1$,

$$
\begin{aligned}
\mathcal{N}_{(m-1)}^{\ell, d}\left[\mathbf{d}^{(m-1)}\right]+ & \mathcal{N}_{(m-1)}^{\ell, a}\left[\mathbf{a}^{(m-1)}\right] \\
& -\mathcal{N}_{(m)}^{u, d}\left[\mathbf{d}^{(m)}\right]-\mathcal{N}_{(m)}^{u, a}\left[\mathbf{a}^{(m)}\right]=\psi^{(m)},
\end{aligned}
$$

and

$$
\mathcal{N}_{(M-1)}^{\ell, d}\left[\mathbf{d}^{(M-1)}\right]+\mathcal{N}_{(M-1)}^{\ell, a}\left[\mathbf{a}^{(M-1)}\right]-\mathcal{N}_{(M)}^{u, d}\left[\mathbf{d}^{(M)}\right]=\psi^{(M)} .
$$

At this point we have $4 M$ equations for $6 M$ unknowns, but we must also remember to enforce the condition that the fields be divergence free which gives $2 M$ more equations. While this is a condition which must be true for all values of $(x, y, z)$ in the bulk, for our purposes it suffices to enforce these at the flat interfaces alone, and thus we define the following operators:

$$
\mathcal{V}(0):\left.\mathbf{a}^{(0)} \rightarrow \operatorname{div}\left[\mathbf{E}^{(0)}\right]\right|_{z=\bar{g}^{(1)}},
$$

and, for $1 \leq m \leq M-1$,

$$
\mathcal{V}(m):\left(\begin{array}{c}
\mathbf{d}^{(m)} \\
\mathbf{a}^{(m)}
\end{array}\right) \rightarrow\left(\begin{array}{c}
\left.\operatorname{div}\left[\mathbf{E}^{(m)}\right]\right|_{z=\bar{g}^{(m)}} \\
\left.\operatorname{div}\left[\mathbf{E}^{(m)}\right]\right|_{z=\bar{g}^{(m+1)}}
\end{array}\right)
$$

and

$$
\mathcal{V}(M):\left.\mathbf{d}^{(M)} \rightarrow \operatorname{div}\left[\mathbf{E}^{(M)}\right]\right|_{z=\bar{g}^{(M)}} .
$$

Once again, introducing helpful notation,

$$
\mathcal{V}(m)=\left(\begin{array}{ll}
\mathcal{V}_{(m)}^{u, d} & \mathcal{V}_{(m)}^{u, a} \\
\mathcal{V}_{(m)}^{\ell, d} & \mathcal{V}_{(m)}^{\ell, a}
\end{array}\right), \quad 1 \leq m \leq M-1,
$$

and

$$
\mathcal{V}(0)=\mathcal{V}_{(0)}^{\ell, a}, \quad \mathcal{V}(M)=\mathcal{V}_{(M)}^{u, d},
$$

our divergence-free conditions take on the form of "boundary conditions,"

$$
\mathcal{V}_{(0)}^{\ell, a}\left[\mathbf{a}^{(0)}\right]=0,
$$

and, for $1 \leq m \leq M-1$,

$$
\mathcal{V}_{(m)}^{u, d}\left[\mathbf{d}^{(m)}\right]+\mathcal{V}_{(m)}^{u, a}\left[\mathbf{a}^{(m)}\right]=0,
$$

$$
\mathcal{V}_{(m)}^{\ell, d}\left[\mathbf{d}^{(m)}\right]+\mathcal{V}_{(m)}^{\ell, a}\left[\mathbf{a}^{(m)}\right]=0,
$$

and

$$
\mathcal{V}_{(M)}^{u, d}\left[\mathbf{d}^{(M)}\right]=0 .
$$

Remark. With all of this notation we note that

$$
\mathcal{D}^{q, r}: \mathcal{F}^{3} \rightarrow \mathcal{F}^{2}, \quad \mathcal{N}^{q, r}: \mathcal{F}^{3} \rightarrow \mathcal{F}^{2}, \quad \mathcal{V}^{q, r}: \mathcal{F}^{3} \rightarrow \mathcal{F},
$$

where $q=\ell, u$ and $r=a, d$.

\section{B. Abstract Formulation}

We state the boundary conditions in Eqs. (3.2) to (3.4) abstractly as

$$
\mathbf{M v}=\mathbf{b},
$$


where

$$
\begin{aligned}
& \mathbf{M}=\left(\begin{array}{lll}
\mathbf{D} & \mathbf{N} & \mathbf{V}
\end{array}\right)^{T}, \\
& \mathbf{v}=\left(\begin{array}{lllllll}
\mathbf{a}^{(0)} & \mathbf{d}^{(1)} & \mathbf{a}^{(1)} & \cdots & \mathbf{d}^{(M-1)} & \mathbf{a}^{(M-1)} & \mathbf{d}^{(M)}
\end{array}\right)^{T}, \\
& \mathbf{b}=\left(\begin{array}{lllllllll}
\zeta^{(1)} & \cdots & \zeta^{(M)} & \psi^{(1)} & \cdots & \psi^{(M)} & 0 & \cdots & 0
\end{array}\right)^{T} .
\end{aligned}
$$

The operators $\mathbf{D}, \mathbf{N}$, and $\mathbf{V}$ clearly require more explanation. In short, they enforce the Dirichlet, Neumann, and divergencefree conditions simultaneously over all layers. For an $M$-layer configuration each of these operators maps

$$
\mathbf{D}, \mathbf{N}, \mathbf{V}: \mathcal{F}^{6 M} \rightarrow \mathcal{F}^{2 M},
$$

and we think of each as a $(2 M) \times(6 M)$ operator-valued matrix. The entireties of these matrices are all zero save the exceptions we detail below (in these we use MATLAB [40] "colon notation" for matrix assignment; for instance, $\mathbf{D}(1: 2,1: 3)$ refers to the $2 \times 3$ matrix with row indices 1 and 2 and column indices 1, 2, and 3). For the $\mathbf{D}$ we have

$$
\begin{aligned}
& \mathbf{D}(2 m-1: 2 m, 6 m-8: 6 m-6)=\mathcal{D}_{(m-1)}^{\ell, d} \quad 2 \leq m \leq M, \\
& \mathbf{D}(2 m-1: 2 m, 6 m-5: 6 m-3)=\mathcal{D}_{(m-1)}^{\ell, a} \quad 1 \leq m \leq M, \\
& \mathbf{D}(2 m-1: 2 m, 6 m-2: 6 m)=-\mathcal{D}_{(m)}^{u, d} \quad 1 \leq m \leq M, \\
& \mathbf{D}(2 m-1: 2 m, 6 m+1: 6 m+3)=-\mathcal{D}_{(m)}^{u, d} \quad 1 \leq m \leq M-1,
\end{aligned}
$$

while for the $\mathbf{N}$ we have

$$
\begin{aligned}
& \mathbf{N}(2 m-1: 2 m, 6 m-8: 6 m-6)=\mathcal{N}_{(m-1)}^{\ell, d} \quad 2 \leq m \leq M, \\
& \mathbf{N}(2 m-1: 2 m, 6 m-5: 6 m-3)=\mathcal{N}_{(m-1)}^{\ell, a} \quad 1 \leq m \leq M, \\
& \mathbf{N}(2 m-1: 2 m, 6 m-2: 6 m)=-\mathcal{N}_{(m)}^{u, d} \quad 1 \leq m \leq M, \\
& \mathbf{N}(2 m-1: 2 m, 6 m+1: 6 m+3)=-\mathcal{N}_{(m)}^{u, d} \quad 1 \leq m \leq M-1 .
\end{aligned}
$$

The operator $\mathbf{V}$ is a little different in structure but has nonzero entries

$$
\begin{aligned}
& \mathbf{V}(2 m, 2 m)=\mathcal{V}_{(m)}^{u, d} \quad 1 \leq m \leq M, \\
& \mathbf{V}(2 m, 2 m+1)=\mathcal{V}_{(m)}^{u, a} \quad 1 \leq m \leq M-1, \\
& \mathbf{V}(2 m+1,2 m)=\mathcal{V}_{(m)}^{\ell, d} \quad 1 \leq m \leq M-1, \\
& \mathbf{V}(2 m+1,2 m+1)=\mathcal{V}_{(m)}^{\ell, a} \quad 0 \leq m \leq M-1 .
\end{aligned}
$$

\section{Taylor Expansions}

The FE approach to this problem is to consider deformations of the form $g^{(m)}(x, y)=\varepsilon f^{(m)}(x, y)\left(f^{(m)}=\mathcal{O}(1)\right)$ and note that, for $f^{(m)}$ sufficiently smooth (Lipschitz) and $\varepsilon$ sufficiently small, the linear operator $\mathbf{M}$ and inhomogeneity $\mathbf{b}$ are both analytic in $\varepsilon$ [41,42]. Furthermore, an analytic solution $\mathbf{v}$ can be shown to exist. More specifically, the following expansions can be demonstrated to be strongly convergent:

$$
\{\mathbf{M}, \mathbf{v}, \mathbf{b}\}(\varepsilon f)=\sum_{n=0}^{\infty}\left\{\mathbf{M}_{n}(f), \mathbf{v}_{n}(f), \mathbf{b}(f)\right\} \varepsilon^{n} .
$$

Crucially, an algorithm for recovering the $\mathbf{v}_{n}$ can be devised based upon regular perturbation theory. In short, we write Eq. (3.5) as

$$
\left(\sum_{n=0}^{\infty} \mathbf{M}_{n} \varepsilon^{n}\right)\left(\sum_{m=0}^{\infty} \mathbf{v}_{m} \varepsilon^{m}\right)=\sum_{n=0}^{\infty} \mathbf{b}_{n} \varepsilon^{n},
$$

and, equating at each perturbation order, we find

$$
\mathbf{M}_{0} \mathbf{v}_{n}=\mathbf{b}_{n}-\sum_{m=0}^{n-1} \mathbf{M}_{n-m} \mathbf{v}_{m} .
$$

At order zero we recover the flat-interface solution, while higher order corrections, $\mathbf{v}_{n}$, can be we computed by appealing to Eq. (3.6). Of great importance is the fact that one only need invert the same linear operator, $\mathbf{M}_{0}$, at every perturbation order. All that remains is a specification of the terms $\left\{\mathbf{M}_{n}, \mathbf{b}_{n}\right\}$; the details can be found at the author's website [43].

To give a flavor of these formulas we consider the uppermost layer. The operator $\mathcal{V}$ is independent of the layer profile shapes $g^{(m)}$, so $\mathcal{V}=\mathcal{V}_{0}$ and $\mathbf{V}=\mathbf{V}_{0}$. In terms of Fourier multiplier notation,

$$
\begin{aligned}
& \mathcal{V}_{0}(0)_{1,1}=i \alpha_{D} \\
& \mathcal{V}_{0}(0)_{1,2}=i \beta_{D}, \\
& \mathcal{V}_{0}(0)_{1,3}=i \gamma_{D}^{(0)} .
\end{aligned}
$$

Regarding the Dirichlet trace operators, upon defining

$$
F_{n}^{(1)}(x, y):=f^{(1)}(x, y)^{n} / n !
$$

one can show that

$$
\begin{aligned}
& \mathcal{D}_{n}(0)_{1,1}=0, \\
& \mathcal{D}_{n}(0)_{1,2}=-F_{n}^{(1)}\left(i \gamma_{D}^{(0)}\right)^{n}, \\
& \mathcal{D}_{n}(0)_{1,3}=-\left(\partial_{y} f^{(1)}\right) F_{n-1}^{(1)}\left(i \gamma_{D}^{(0)}\right)^{n-1},
\end{aligned}
$$

and

$$
\begin{aligned}
& \mathcal{D}_{n}(0)_{2,1}=F_{n}^{(1)}\left(i \gamma_{D}^{(0)}\right)^{n}, \\
& \mathcal{D}_{n}(0)_{2,2}=0, \\
& \mathcal{D}_{n}(0)_{2,3}=\left(\partial_{x} f^{(1)}\right) F_{n-1}^{(1)}\left(i \gamma_{D}^{(0)}\right)^{n-1} .
\end{aligned}
$$

Finally, for the Neumann trace operators,

$$
\begin{aligned}
& \mathcal{N}_{n}(0)_{1,1}=-F_{n}^{(1)}\left(i \gamma_{D}^{(0)}\right)^{n+1}+\left(\partial_{y} f^{(1)}\right) F_{n-1}^{(1)}\left(i \beta_{D}\right)\left(i \gamma_{D}^{(0)}\right)^{n-1}, \\
& \mathcal{N}_{n}(0)_{1,2}=-\left(\partial_{y} f^{(1)}\right) F_{n-1}^{(1)}\left(i \alpha_{D}\right)\left(i \gamma_{D}^{(0)}\right)^{n-1}, \\
& \mathcal{N}_{n}(0)_{1,3}=F_{n}^{(1)}\left(i \alpha_{D}\right)\left(i \gamma_{D}^{(0)}\right)^{n},
\end{aligned}
$$

and

$$
\begin{aligned}
& \mathcal{N}_{n}(0)_{2,1}=-\left(\partial_{x x} f^{(1)}\right) F_{n-1}^{(1)}\left(i \beta_{D}\right)\left(i \gamma_{D}^{(0)}\right)^{n-1}, \\
& \mathcal{N}_{n}(0)_{2,2}=-F_{n}^{(1)}\left(i \gamma_{D}^{(0)}\right)^{n+1}+\left(\partial_{x} f^{(1)}\right) F_{n-1}^{(1)}\left(i \alpha_{D}\right)\left(i \gamma_{D}^{(0)}\right)^{n-1}, \\
& \mathcal{N}_{n}(0)_{2,3}=F_{n}^{(1)}\left(i \beta_{D}\right)\left(i \gamma_{D}^{(0)}\right)^{n} .
\end{aligned}
$$

\section{NUMERICAL RESULTS}

We now present results of numerical simulations conducted with the generalized FE method outlined above. The scheme can be viewed as a high-order spectral approach where nonlinearities are approximated with convolutions implemented via the fast Fourier transform algorithm [44]. 


\section{A. Exact Solutions}

Following the developments of several of our previous papers on similar topics (see, e.g., $[45,46]$ ), we consider the functions

$$
\mathbf{E}_{r, s}^{(0)}(x, y, z)=\mathbf{a}_{r, s}^{(0)} e^{i \gamma_{r, s}^{(0)}\left(z-\bar{g}^{(1)}\right)} e^{i\left(\alpha_{r} x+\beta_{s} y\right)}
$$

and, for $1 \leq m \leq M$,

$$
\begin{aligned}
\mathbf{E}_{r, s}^{(m)}(x, y, z)= & \left\{\mathbf{d}_{r, s}^{(m)} \frac{\sinh \left(i \gamma_{r, s}^{(m)}\left(z-\bar{g}^{(m+1)}\right)\right)}{\sinh \left(i \gamma_{r, s}^{(m)}\left(\bar{g}^{(m)}-\bar{g}^{(m+1)}\right)\right)}\right. \\
& \left.+\mathbf{a}_{r, s}^{(m)} \frac{\sinh \left(i \gamma_{r, s}^{(m)}\left(\bar{g}^{(m)}-z\right)\right)}{\sinh \left(i \gamma_{r, s}^{(m)}\left(\bar{g}^{(m)}-\bar{g}^{(m+1)}\right)\right)}\right\} e^{i\left(\alpha_{r} x+\beta_{s} y\right)},
\end{aligned}
$$

and

$$
\mathbf{E}_{r, s}^{(M)}(x, y, z)=\mathbf{d}_{r, s}^{(M)} e^{i \gamma_{r, s}^{(M)}\left(\bar{g}^{(M)}-z\right)} e^{i\left(\alpha_{r} x+\beta_{s} y\right)},
$$

with $(r, s) \in \mathbf{Z}^{2}$. These are outgoing, quasiperiodic solutions of the Helmholtz equation. However, the boundary conditions satisfied by these functions are not those satisfied by an incident plane wave. We compute the surface data

$$
\begin{aligned}
\tilde{\zeta}^{(m)} & :=N^{(m)} \times\left[\mathbf{E}_{r, s}^{(m-1)}-\mathbf{E}_{r, s}^{(m)}\right]_{z=\bar{g}^{(m)}+g^{(m)}}, \\
\tilde{\psi}^{(m)} & :=N^{(m)} \times\left[\nabla \times\left[\mathbf{E}_{r, s}^{(m-1)}-\mathbf{E}_{r, s}^{(m)}\right]\right]_{z=\bar{g}^{(m)}+g^{(m)}},
\end{aligned}
$$

for $1 \leq m \leq M$. This is a family of exact solutions against which to test our numerical algorithm for any choice of deformations $\left\{g^{(1)}, \ldots, g^{(M)}\right\}$.

\section{B. Implementation and Error Measurement}

The method described in Section 3 is essentially a Fourier collocation [44]/Taylor method [36,47] enhanced by a Padé approximation $[\underline{42}, \underline{48}]$. More specifically, we approximate the fields, $\left\{\mathbf{E}^{(0)}, \overline{\mathbf{E}^{(m)}}, \mathbf{E}^{(M)}\right\}$, by

$$
\mathbf{E}^{\left(0, N_{x}, N_{y}, N\right)}:=\sum_{n=0}^{N} \sum_{p=-N_{x} / 2}^{N_{x} / 2-1} \sum_{q=-N_{y} / 2}^{N_{y} / 2-1} \mathbf{a}_{p, q}^{(0)} e^{i \gamma_{p, q}^{(0)}\left(z-\bar{g}^{(1)}\right)} e^{i\left(\alpha_{p} x+\beta_{q} y\right)} \varepsilon^{n},
$$

and, for $1 \leq m \leq M-1$,

$$
\begin{aligned}
\mathbf{E}^{\left(m, N_{x}, N_{y}, N\right)}:= & \sum_{n=0}^{N} \sum_{p=-N_{x} / 2}^{N_{x} / 2-1} \sum_{q=-N_{y} / 2}^{N_{y} / 2-1} \\
& \times\left\{\mathbf{d}_{p, q}^{(m)} \frac{\sinh \left(i \gamma_{p, q}^{(m)}\left(z-\bar{g}^{(m+1)}\right)\right)}{\sinh \left(i \gamma_{p, q}^{(m)}\left(\bar{g}^{(m)}-\bar{g}^{(m+1)}\right)\right)}\right. \\
& \left.+\mathbf{a}_{p, q}^{(m)} \frac{\sinh \left(i \gamma_{p, q}^{(m)}\left(\bar{g}^{(m)}-z\right)\right)}{\sinh \left(i \gamma_{p, q}^{(m)}\left(\bar{g}^{(m)}-\bar{g}^{(m+1)}\right)\right)}\right\} e^{i\left(\alpha_{p} x+\beta_{q} y\right)} \varepsilon^{n},
\end{aligned}
$$

and

$$
\mathbf{E}^{\left(M, N_{x}, N_{y}, N\right)}:=\sum_{n=0}^{N} \sum_{p=-N_{x} / 2}^{N_{x} / 2-1} \sum_{q=-N_{y} / 2}^{N_{y} / 2-1} \mathbf{d}_{p, q}^{(M)} e^{i \gamma_{p, q}^{(M)}\left(\bar{g}^{(M)}-z\right)} e^{i\left(\alpha_{p} x+\beta_{q} y\right)} \varepsilon^{n},
$$

respectively [cf. Eq. (2.4)]. We insert these into Eq. (3.6) and determine the $\left\{\mathbf{v}_{n}\right\}$.

A crucial consideration is how the Taylor series in $\varepsilon$ are summed. To be specific, to approximate $\mathbf{E}^{(0)}$ we consider the truncation $\mathbf{E}^{\left(0, N_{x}, N_{y}, N\right)}$, which amounts to the approximation $\mathbf{a}_{p, q}(\varepsilon):=\sum_{n=0}^{\infty} \mathbf{a}_{p, q}^{(0)} \varepsilon^{n}$ by $\mathbf{a}_{p, q}^{N}(\varepsilon):=\sum_{n=0}^{N} \mathbf{a}_{p, q}^{(0)} \varepsilon^{n}$. The classical numerical analytic continuation technique of Padé approximation [48] has been successfully brought to bear upon HOPS methods in the past (see, e.g., [34,42]), and we advocate its use here. The Padé approximation seeks to simulate the truncated Taylor series $\mathbf{a}_{p, q}^{N}(\varepsilon)$ by the rational function

$$
[L / M](\varepsilon):=\frac{a^{L}(\varepsilon)}{b^{M}(\varepsilon)}=\frac{\sum_{\ell=0}^{L} a_{\ell} \varepsilon^{\ell}}{1+\sum_{m=1}^{M} b_{m} \varepsilon^{m}},
$$

where $L+M=N$ and $[L / M](\varepsilon)=\mathbf{a}_{p, q}^{N}(\varepsilon)+\mathcal{O}\left(\varepsilon^{L+M+1}\right)$; wellknown formulas for the coefficients $\left\{a_{\ell}, b_{m}\right\}$ can be found in [48]. This approximant has remarkable properties of enhanced convergence, and we refer the interested reader to Section 2.2 of Baker and Graves-Morris [48] and the insightful calculations of Section 8.3 of Bender and Orszag [49] for a thorough discussion of the capabilities and limitations of Padé approximants.

With these approximations we can now measure error in our simulations, Eq. (4.2), versus the exact solutions, Eq. (4.1). Among the myriad choices available we select the relative defect in the top and bottom Dirichlet data measured in the supremum norm,

$$
\text { Error }:=\max \left\{\frac{\left|\mathbf{E}^{\left(0, N_{x}, N_{y}, N\right)}-\mathbf{E}^{(0)}\right|_{L^{\infty}}}{\left|\mathbf{E}^{(0)}\right|_{L^{\infty}}}, \frac{\left|\mathbf{E}^{\left(M, N_{x}, N_{y}, N\right)}-\mathbf{E}^{(M)}\right|_{L^{\infty}}}{\left|\mathbf{E}^{(M)}\right|_{L^{\infty}}}\right\} .
$$

\section{Convergence Study}

For our convergence study we select the doubly $2 \pi$ periodic profiles $g^{(m)}=\varepsilon f^{(1)}$,

$$
\begin{aligned}
& f^{(1)}(x, y)=\cos (x)+\cos (y), \\
& f^{(2)}(x, y)=\cos (2 x)+\cos (2 y),
\end{aligned}
$$

and the physical and numerical parameters

$$
\begin{array}{r}
\alpha=0.1, \quad \beta=0.2, \quad \gamma=(1.1,2.2,3.3)^{T}, \\
\varepsilon=0.003,0.01,0.03,0.1, \quad d_{1}=d_{2}=2 \pi, \\
N_{x}=N_{y}=24, \quad N=0, \ldots, 10 .
\end{array}
$$

In Figs. $\underline{1}$ and $\underline{2}$ we summarize the results of these simulations which not only demonstrate the validity of our codes but also show that only a small number of Fourier modes and perturbation orders are required to realize machine precision 


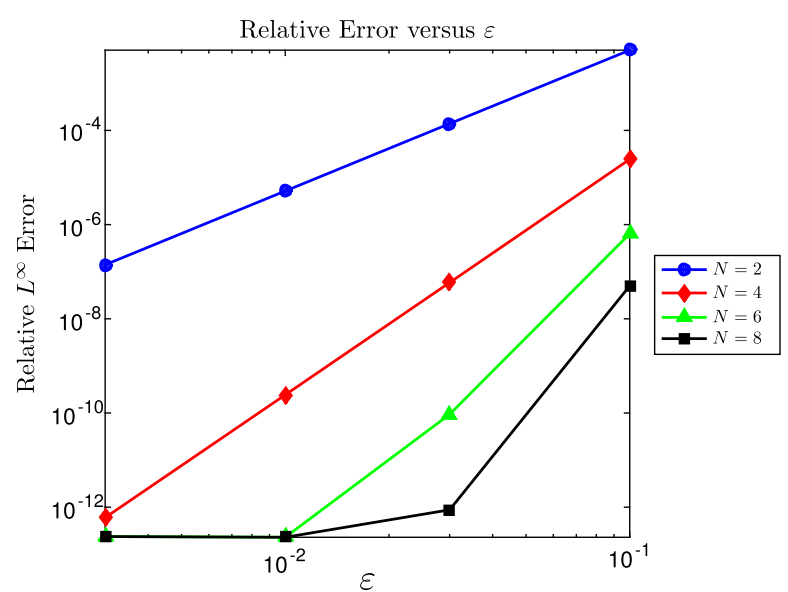

Fig. 1. Relative error versus perturbation parameter $\varepsilon$ for various perturbation orders $N$ (with $[N / 2, N / 2]$ Padé approximation). Results for the sinusoid-sinusoid configuration, Eq. (․4), with $N_{x}=N_{y}=24$.

(up to the conditioning of the algorithm) for small (e.g., $\varepsilon=0.01$ ), smooth profiles.

\section{SPR Simulations}

To close, we consider a configuration which is not substantially different from one recently considered in the laboratory of Oh (Minnesota), in particular the devices described in $[\underline{12}, \underline{15}]$. In these latter publications a two-dimensional thinfilm sensor was investigated which was corrugated on one side and flat on the other. With the current computational capability we can investigate with great ease not only the presence of corrugations on both sides but also full threedimensionality.

To begin, we consider a reference configuration consisting of two layers: vacuum (a dielectric) above gold (a metal), separated by the perturbed interface $g^{(1)}=h f^{(1)}$,

$$
f^{(1)}(x, y)=\frac{1}{4}\left\{\cos \left(2 \pi x / d_{1}\right)+\cos \left(2 \pi y / d_{2}\right)\right\} ;
$$

without loss of generality we set $\bar{g}^{(1)}=0$. By definition, the refractive index for vacuum is $n^{v}=1$. The refractive index

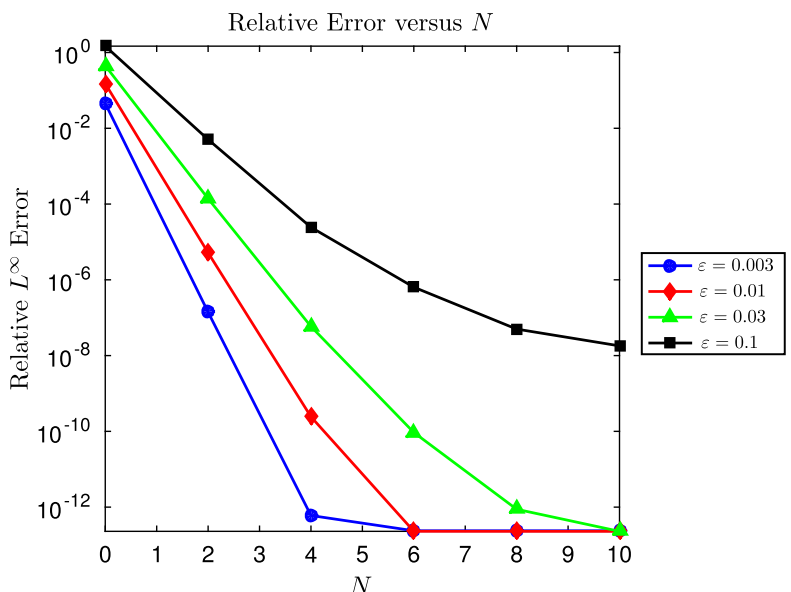

Fig. 2. Relative error versus perturbation order $N$ (with $[N / 2, N / 2]$ Padé approximation) for various perturbation parameters $\varepsilon$. Results for the sinusoid-sinusoid configuration, Eq. (4.4), with $N_{x}=N_{y}=24$.

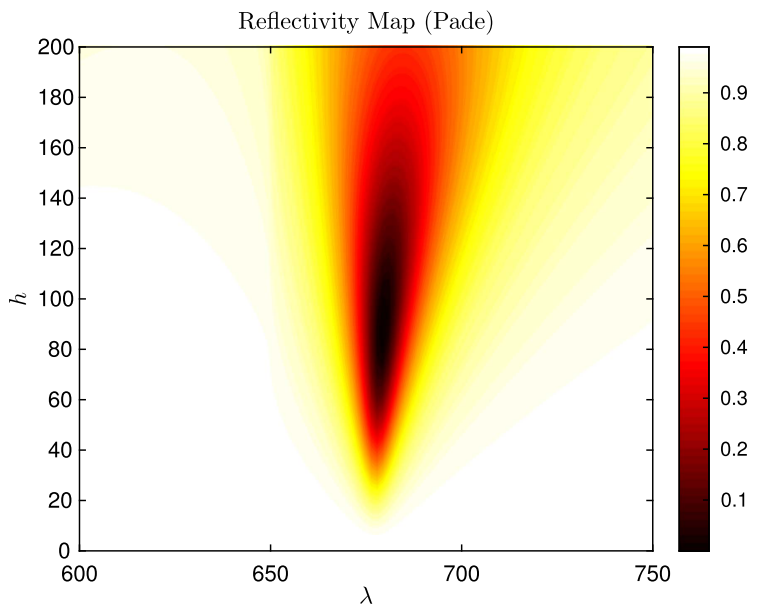

Fig. 3. Reflectivity map for two layers, $R(\lambda, h)$, versus incident wavelength, $\lambda$, and deformation height, $h$. Results for the sinusoid configuration, Eq. (4.5), with $N_{x}=N_{y}=24$, [8/8] Padé approximant.

of gold is the subject of ongoing research, and we choose a Lorentz model [50],

$$
\epsilon^{A u}=\epsilon_{\infty}^{A u}+\sum_{j=1}^{6} \frac{\Delta_{j}^{A u}}{-a_{j}^{A u} \omega^{2}-i b_{j}^{A u} \omega+c_{j}^{A u}},
$$

where $\omega=2 \pi / \lambda, \epsilon_{\infty}^{A u}=1$, and $\Delta^{A u}, a^{A u}, b^{A u}$, and $c^{A u}$ can be found in [50]. For physical and numerical parameters we select the following:

$$
\begin{gathered}
\alpha=0, \quad \beta=0, \quad \gamma=\left(\gamma^{v}, \gamma^{A u}\right)^{T}, \\
h=0, \ldots, 0.2, \quad d_{1}=d_{2}=0.650, \\
N_{x}=N_{y}=24, \quad N=0, \ldots, 16 .
\end{gathered}
$$

In Fig. 3 we display the reflectivity map for this configuration, which shows a strong plasmonic response around $\lambda=0.680$ and $h=0.080$ (both measured in micrometers).

By comparison we consider the three-layer configuration composed of vacuum (dielectric) overlaying a thin layer of gold (metal) of thickness $t$ on top of water (dielectric) with interfaces shaped by $g^{(m)}=h f^{(m)}$,

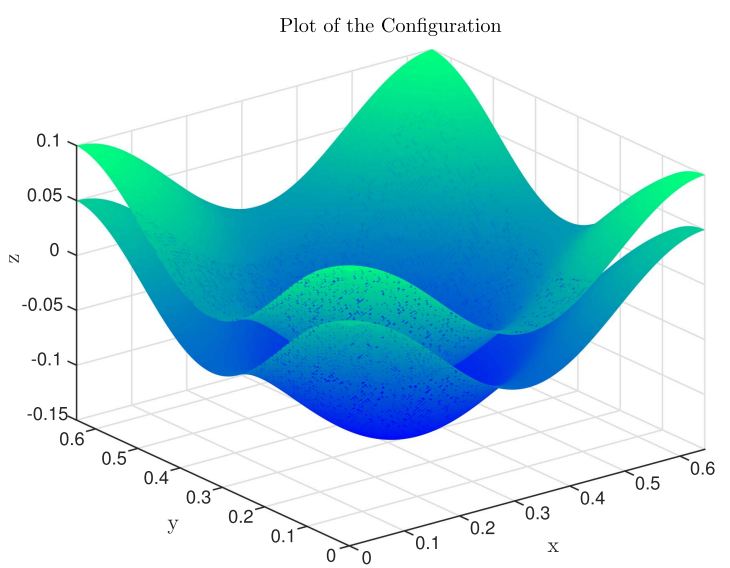

Fig. 4. Plot of the three-layer configuration Eq. (4) with $t=50 \mathrm{~nm}$. 


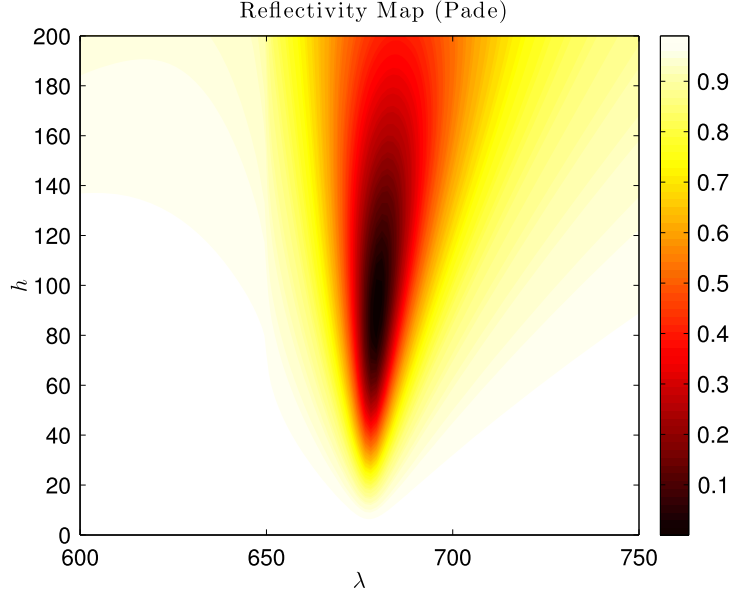

Fig. 5. Reflectivity map for three layers, $R(\lambda, h)$, versus incident wavelength, $\lambda$, and deformation height, $h$. Results for gold layer thickness $t=100 \mathrm{~nm}$ and the double sinusoid configuration, Eq. (뜨), with $N_{x}=N_{y}=24$, [8/8] Padé approximant.

$$
f^{(1)}(x, y)=f^{(2)}(x, y)=\frac{1}{4}\left\{\cos \left(2 \pi x / d_{1}\right)+\cos \left(2 \pi y / d_{2}\right)\right\}
$$

see Fig. 4 . We set $\bar{g}^{(1)}=0$ and $\bar{g}^{(2)}=-t(t=50,100 \mathrm{~nm})$ and use the refractive index of water, $n^{w}=1.333$ [12]. For physical and numerical parameters we select the following:

$$
\begin{array}{r}
\alpha=0, \quad \beta=0, \quad \gamma=\left(\gamma^{v}, \gamma^{A u}, \gamma^{w}\right)^{T}, \\
h=0, \ldots, 0.2, \quad d_{1}=d_{2}=0.650 \\
N_{x}=N_{y}=24, \quad N=0, \ldots, 16 .
\end{array}
$$

We point out that while the reflectivity map for the $t=$ $100 \mathrm{~nm}$ configuration (see Fig. 5) looks qualitatively very similar to the semi-infinite layer case depicted in Fig. 3, the reflectivity map for $t=50 \mathrm{~nm}$ (see Fig. 6) looks completely

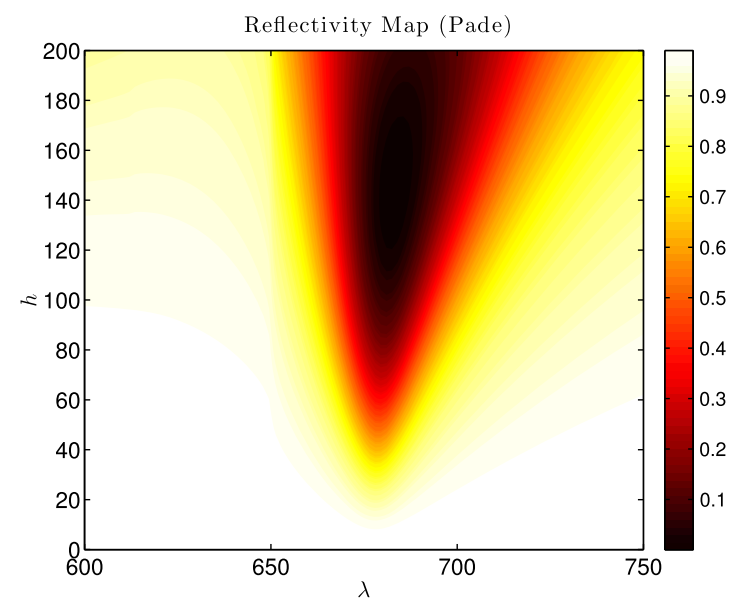

Fig. 6. Reflectivity map for three layers, $R(\lambda, h)$, versus incident wavelength, $\lambda$, and deformation height, $h$. Results for gold layer thickness $t=50 \mathrm{~nm}$ and the double sinusoid configuration, Eq. (4.6), with $N_{x}=N_{y}=24$, [8/8] Padé approximant. different as the region of sensitive response is vastly enlarged. One factor for this difference is the fact that the thin-layer configuration allows radiation to transmit into the water as its vertical dimension is now comparable to the skin depth of gold. The point of our contribution is that with our new methodology it is very simple to vary such configuration parameters and evaluate a robust and reliable simulation in a matter of a minute or two on a laptop computer.

\section{REFERENCES}

1. J. Virieux and S. Operto, "An overview of full-waveform inversion in exploration geophysics," Geophysics 74, WCC1-WCC26 (2009).

2. L. M. Brekhovskikh and Y. P. Lysanov, Fundamentals of Ocean Acoustics (Springer-Verlag, 1982).

3. C. Godrèche, ed., Solids Far from Equilibrium (Cambridge University, 1992)

4. F. Natterer and F. Wübbeling, "Mathematical methods in image reconstruction," in SIAM Monographs on Mathematical Modeling and Computation (Society for Industrial and Applied Mathematics [SIAM], 2001).

5. H. Raether, Surface Plasmons on Smooth and Rough Surfaces and on Gratings (Springer, 1988).

6. S. Maier, Plasmonics: Fundamentals and Applications (Springer, 2007).

7. S. Enoch and N. Bonod, Plasmonics: From Basics to Advanced Topics, Springer Series in Optical Sciences (Springer, 2012).

8. T. Ebbesen, H. Lezec, H. Ghaemi, T. Thio, and P. Wolff, "Extraordinary optical transmission through sub-wavelength hole arrays," Nature 391, 667-669 (1998).

9. M. Moskovits, "Surface-enhanced spectroscopy," Rev. Mod. Phys. 57, 783-826 (1985).

10. J. Homola, "Surface plasmon resonance sensors for detection of chemical and biological species," Chem. Rev. 108, 462-493 (2008)

11. H. Im, S. H. Lee, N. J. Wittenberg, T. W. Johnson, N. C. Lindquist, P. Nagpal, D. J. Norris, and S. H. Oh, "Template-stripped smooth Ag nanohole arrays with silica shells for surface plasmon resonance biosensing," ACS Nano 5, 6244-6253 (2011).

12. N. C. Lindquist, T. W. Johnson, J. Jose, L. M. Otto, and S.-H. Oh, "Ultrasmooth metallic films with buried nanostructures for backside reflection-mode plasmonic biosensing," Ann. Phys. 524, 687-696 (2012).

13. J. Jose, L. Jordan, T. Johnson, S. Lee, N. Wittenberg, and S. Oh, "Topographically flat substrates with embedded nanoplasmonic devices for biosensing," Adv. Funct. Mater. 23, 2812-2820 (2013).

14. F. Reitich, T. Johnson, S.-H. Oh, and G. Meyer, "A fast and highorder accurate boundary perturbation method for characterization and design in nanoplasmonics," J. Opt. Soc. Am. A 30, 21752187 (2013).

15. D. P. Nicholls, F. Reitich, T. Johnson, and S.-H. Oh, "Fast highorder perturbation of surfaces (HOPS) methods for simulation of multi-layer plasmonic devices and metamaterials," J. Opt. Soc. Am. A 31, 1820-1831 (2014).

16. A. Schadle, L. Zschiedrich, S. Burger, R. Klose, and F. Schmidt, "Domain decomposition method for Maxwell's equations: scattering off periodic structures," J. Comput. Phys. 226, 477-493 (2007).

17. G. Demesy, F. Zolla, A. Nicolet, and M. Commandre, "Versatile full-vectorial finite element model for crossed gratings," Opt. Lett. 34, 2216-2219 (2009).

18. M. Huber, J. Schoberl, A. Sinwel, and S. Zaglmayr, "Simulation of diffraction in periodic media with a coupled finite element and plane wave approach," SIAM J. Sci. Comput. 31, 1500-1517 (2009).

19. K. Stannigel, M. Konig, J. Niegemann, and K. Busch, "Discontinuous Galerkin time-domain computations of metallic nanostructures," Opt. Express 17, 14934-14947 (2009).

20. D. Christensen and D. Fowers, "Modeling SPR sensors with the finite-difference time-domain method," Biosens. Bioelectron 11, 677-684 (1996). 
21. H. Sai, Y. Kanamori, K. Hane, and H. Yugami, "Numerical study on spectral properties of Tungsten one-dimensional surfacerelief gratings for spectrally selective devices," J. Opt. Soc. Am. A 22, 1805-1813 (2005).

22. N. Lindquist, T. Johnson, D. Norris, and S.-H. Oh, "Monolithic integration of continuously tunable plasmonic nanostructures," Nano Lett. 11, 3526-3530 (2011).

23. J.-P. Berenger, "A perfectly matched layer for the absorption of electromagnetic waves," J. Comput. Phys. 114, 185-200 (1994).

24. D. Givoli, "Nonreflecting boundary conditions," J. Comput. Phys. 94, 1-29 (1991).

25. D. Givoli, "Numerical methods for problems in infinite domains," in Studies in Applied Mechanics, Vol. 33 (Elsevier Scientific Publishing Co., 1992).

26. D. Givoli, "Recent advances in the DtN FE method," Arch. Comput. Methods Eng. 6, 71-116 (1999).

27. D. Colton and R. Kress, Inverse Acoustic and Electromagnetic Scattering Theory, 2nd ed. (Springer-Verlag, 1998).

28. L. Greengard and V. Rokhlin, "A fast algorithm for particle simulations," J. Comput. Phys. 73, 325-348 (1987).

29. F. Reitich and K. Tamma, "State-of-the-art, trends, and directions in computational electromagnetics," Comput. Model. Eng. Sci. 5, 287-294 (2004).

30. H. Kurkcu and F. Reitich, "Stable and efficient evaluation of periodized Green's functions for the Helmholtz equation at high frequencies," J. Comput. Phys. 228, 75-95 (2009).

31. L. Rayleigh, "On the dynamical theory of gratings," Proc. R. Soc. London 79, 399-416 (1907).

32. S. O. Rice, "Reflection of electromagnetic waves from slightly rough surfaces," Commun. Pure Appl. Math. 4, 351-378 (1951).

33. O. P. Bruno and F. Reitich, "Numerical solution of diffraction problems: a method of variation of boundaries," J. Opt. Soc. Am. A 10, 1168-1175 (1993).

34. O. P. Bruno and F. Reitich, "Numerical solution of diffraction problems: a method of variation of boundaries. II. Finitely conducting gratings, Padé approximants, and singularities," J. Opt. Soc. Am. A 10, 2307-2316 (1993).

35. O. P. Bruno and F. Reitich, "Numerical solution of diffraction problems: a method of variation of boundaries. III. Doubly periodic gratings," J. Opt. Soc. Am. A 10, 2551-2562 (1993).
36. D. P. Nicholls and F. Reitich, "Shape deformations in rough surface scattering: cancellations, conditioning, and convergence," J. Opt. Soc. Am. A 21, 590-605 (2004).

37. D. P. Nicholls and F. Reitich, "Shape deformations in rough surface scattering: improved algorithms," J. Opt. Soc. Am. A 21, 606-621 (2004)

38. D. P. Nicholls and F. Reitich, "Boundary perturbation methods for high-frequency acoustic scattering: shallow periodic gratings," J. Acoust. Soc. Am. 123, 2531-2541 (2008).

39. A. Malcolm and D. P. Nicholls, "A field expansions method for scattering by periodic multilayered media," J. Acoust. Soc. Am. 129, 1783-1793 (2011).

40. MATLAB, version 7.10.0 (R2010a) (The MathWorks Inc., 2010).

41. D. P. Nicholls and F. Reitich, "A new approach to analyticity of Dirichlet-Neumann operators," Proc. R. Soc. Edinburgh Sect. A 131, 1411-1433 (2001).

42. D. P. Nicholls and F. Reitich, "Analytic continuation of DirichletNeumann operators," Numer. Math. 94, 107-146 (2003).

43. www.math.uic.edu/ nicholls.

44. D. Gottlieb and S. A. Orszag, Numerical Analysis of Spectral Methods: Theory and Applications, Vol. 26 of CBMS-NSF Regional Conference Series in Applied Mathematics (Society for Industrial and Applied Mathematics, 1977).

45. D. Ambrose and D. P. Nicholls, "Fokas integral equations for three dimensional layered-media scattering," J. Comput. Phys. 276, 1-25 (2014)

46. D. P. Nicholls, "A high-order perturbation of surfaces (HOPS) approach to Fokas integral equations: three-dimensional layered media scattering," Quart. Appl. Math. (to be published).

47. D. P. Nicholls and F. Reitich, "Stability of high-order perturbative methods for the computation of Dirichlet-Neumann operators," J. Comput. Phys. 170, 276-298 (2001).

48. G. A. Baker, Jr. and P. Graves-Morris, Padé Approximants, 2nd ed. (Cambridge University, 1996).

49. C. M. Bender and S. A. Orszag, Advanced Mathematical Methods for Scientists and Engineers, International Series in Pure and Applied Mathematics (McGraw-Hill, 1978).

50. A. Rakic, A. Djurisic, J. Elazar, and M. Majewski, "Optical properties of metallic films for vertical-cavity optoelectronic devices," Appl. Opt. 37, 5271-5283 (1998). 